\title{
Cascading Pressure Thermal Reduction for Efficient Solar Fuel Production
}

\author{
Ivan Ermanoski \\ Sandia National Laboratories, Albuquerque, NM 87185; iermano@sandia.gov
}

\begin{abstract}
Efficient two-step solar-thermochemical fuel production requires vacuum pumping or inert gas sweeping to lower the oxygen pressure in the thermal reduction step. Pumping is hampered by large oxygen volumetric flows, whereas sweeping is energy-intensive, requiring heat recovery at high temperature, and a dedicated inert gas purification plant. A novel pumping approachusing a cascade of chambers at successively lower pressures - is analyzed and shown to lead to over an order of magnitude pressure decrease compared to a single-chambered design. The resulting efficiency gains are substantial, and represent an important step toward practical and efficient solar fuel production on a large scale.
\end{abstract}

Keywords: Hydrogen, thermochemical, solar fuels, carbon dioxide

\section{Introduction}

Two-step thermochemical cycles are a conceptually simple, theoretically highly efficient, and promising approach for solar fuel production. In the first step - thermal reduction - a reactive material (oxide) is partially or fully reduced at high temperature (typically 1600-1800K). In the second step-fuel production-the reduced oxide is exposed to steam or $\mathrm{CO}_{2}$ at a lower temperature (1100-1500K), to produce $\mathrm{H}_{2}$ or CO.[1-4]

Efficiencies achieved in experiments have been much lower than theoretical limits, largely owing to the performance of existing reactive oxides. The low reversible oxygen capacity of these oxides results in low $\mathrm{H}_{2}$ or $\mathrm{CO}$ yields per mole oxide per cycle, leading to large energy requirements for oxide heating.[5, 6] Increasing the per-cycle yield has proven to be challenging-requiring high thermal reduction temperatures $\left(T_{T R}\right)$ and low thermal reduction pressures $\left(p_{T R}\right)$. High temperatures cause excessive aperture radiation losses and require the use of specialized construction materials, limiting $T_{T R}$ to $<1800 \mathrm{~K}$. Low pressures can be achieved by inert gas sweeping or vacuum pumping - both facing limitations for reasonably-scaled commercial systems (1-10MW). Sweeping requires large amounts of high-purity gasses and extensive high-temperature heat recovery, whereas pumping requires excessively large vacuum pumps, limiting the minimum achievable $p_{T R \cdot}$ [7]

A previously unexplored pumping approach is the use of a pressure cascade-i.e. multiple thermal reduction chambers at successively lower pressures - to achieve low $p_{T R}$. Theoretical aspects, efficiency benefits, and implementation modes and limits of a pressure cascade, are examined in this paper. The results indicate a potential for a significant $p_{T R}$ limit decrease and efficiency gains in two-step thermochemical cycles.

\section{Thermochemical cycles: vacuum pumping advantages and limitations}

Reactions (1) and (2) below, generically describe a two-step thermochemical cycle for $\mathrm{H}_{2}$ production, based on a reactive metal oxide $\left(\mathrm{MO}_{\mathrm{x}}\right)$. Reaction (1) is an endothermic thermal reduction of the oxide carried out at a temperature $T_{T R}$. Reaction (2) is the mildly exothermic reoxidation of the reduced oxide with $\mathrm{H}_{2} \mathrm{O}$, at temperature $T_{W S}$, which yields $\mathrm{H}_{2}$ and restores the 
oxide to its initial state. The process can also be used to produce $\mathrm{CO}$ from $\mathrm{CO}_{2}$ in reaction (2). The sum of the two reactions is heat-driven $\mathrm{H}_{2} \mathrm{O}$ splitting, described by reaction (3):

$$
\begin{array}{ll}
\frac{1}{\delta_{T R}-\delta_{W S}} M O_{x-\delta_{W S}} \rightarrow \frac{1}{\delta_{T R}-\delta_{W S}} M O_{x-\delta_{T R}}+\frac{1}{2} O_{2} & \text { (1) thermal reduction at } T_{T R}, p_{T R}, \\
\frac{1}{\delta_{T R}-\delta_{W S}} M O_{x-\delta_{T R}}+H_{2} \mathrm{O} \rightarrow \frac{1}{\delta_{T R}-\delta_{W S}} M O_{x-\delta_{W S}}+H_{2} & \text { (2) } \mathrm{H}_{2} \mathrm{O} \text { splitting at } T_{W S}, \\
\mathrm{H}_{2} \mathrm{O} \rightarrow \mathrm{H}_{2}+\frac{1}{2} \mathrm{O}_{2} & \text { (3) } \mathrm{H}_{2} \mathrm{O} \text { thermolysis. }
\end{array}
$$

Here, $\delta_{T R}$ and $\delta_{W S}$ are the extents of reduction of the oxide following the thermal reduction and water splitting steps. Low $p_{T R}$ (or, more generally, $p_{O 2}$ ) in reaction (1) increases $\delta_{T R}$ and the reversible oxygen capacity $\Delta \delta=\delta_{T R}-\delta_{W S}$. Because the $\mathrm{H}_{2}$ yield per mole oxide per cycle is proportional to $\Delta \delta$, lowering $p_{T R}$ generally increases efficiency.[7-12]

Achieving low $p_{T R}$, however advantageous, is challenging. The main difficulty are large $\mathrm{O}_{2}$ volumetric flows - not pumping work, $\mathrm{O}_{2}$ mass flow, or aerodynamic loads.[7] Indeed, a $1 \mathrm{MW}$ chemical output corresponds to $\sim 3.5 \mathrm{~mol} / \mathrm{s} \mathrm{H}_{2}$ and an $\mathrm{O}_{2}$ mass flow rate of only $\sim 55 \mathrm{~g} / \mathrm{s}$. Nonetheless, at low pressure, even modest mass flow rates require pumping at large volumetric rates, $\dot{V}_{O_{2}}$, and at high $\mathrm{O}_{2}$ flow velocities, $v_{O_{2}}=\dot{V}_{O_{2}} / A_{o x}$.

To quantify $v_{O_{2}}$ and $\dot{V}_{O_{2}}$, consider the surface area of the oxide receiver $\left(A_{o x}\right)$, illustrated in the inset in Fig.1a:

$$
A_{\text {ox }}=A_{\text {coll }} / C_{B}
$$

Here $A_{\text {coll }}$ is the solar collector/concentrator area (e.g. gross heliostat area) and $C_{B}$ is the concentration ratio at the oxide receiver (roughly $10 \%$ of the concentration ratio at the aperture, $\left.C_{A}\right)$. The chemical output power, $P$, of a $\mathrm{H}_{2}$-producing reactor, can be expressed in two ways:

$$
P=\eta A_{\text {coll }} \dot{Q}_{S} \quad \text { and } P=\dot{n}_{H_{2}} H H V_{H 2} \quad \text { (5) and (6). }
$$

Here $\eta$ is the reactor solar-to-hydrogen efficiency, $\dot{Q}_{S}=1 \mathrm{~kW} / \mathrm{m}^{2}$ is the incoming solar flux, $\dot{n}_{\mathrm{H}_{2}}$ is the molar $\mathrm{H}_{2}$ production rate, and $H H V_{H 2}=286 \mathrm{~kJ} / \mathrm{mol}$ is the $\mathrm{H}_{2}$ higher heating value. Combining eqs. (4), (5), (6), and the ideal gas equation, the required $v_{O_{2}}$ can be expressed as:

$$
v_{O_{2}}=\frac{\eta C_{B} R T_{T R} * \dot{Q}_{S}}{2 * H H V_{H 2}} \frac{1}{p_{T R}}
$$

Fig.1a shows $v_{O_{2}}$ as function of $p_{T R}$, calculated using eq.(7). While $v_{O_{2}}$ is quite reasonable for $p_{T R} \approx 100 \mathrm{~Pa}$ and higher, it reaches hypersonic and even orbital velocity below $1 \mathrm{~Pa}$. For lower values of $C_{B}$-within a reasonable range- $v_{O_{2}}$ would be somewhat lower than in Fig.1a, but still excessive at low $p_{T R}$.

Assuming a maximum $v_{\mathrm{O}_{2}} \approx 100 \mathrm{~m} / \mathrm{s}$, the lowest feasible $p_{T R}$ is around $20 \mathrm{~Pa}$-illustrating the limits of pumping. Somewhat higher $v_{\mathrm{O}_{2}}$ values could be considered, but with caution regarding practical flow impediments, such as conductance losses in pumping elements and decreased conductance under molecular flow dynamics at low pressures $\left(p_{T R} \lesssim 1 \mathrm{~Pa}\right)$. The speed of sound in $\mathrm{O}_{2}(c \approx 800 \mathrm{~m} / \mathrm{s}$ at $1773 \mathrm{~K})$ is an upper practical limit under all circumstances. 
To calculate $\dot{V}_{O_{2}}$, a normalization with respect to input solar power $\left(P_{S}\right)$ is needed. From $C_{B}=300$ follows $A_{o x} \approx 3.33 \mathrm{~m}^{2} / \mathrm{MW}$, corresponding to $\dot{V}_{O_{2}}(1 P a) \approx \frac{6450 \mathrm{~m}^{3}}{\mathrm{~s}} / M W$ at $\eta=25 \%$. This impractically large value further underscores the challenges of vacuum pumping. To illustrate the advantages of low $p_{T R}$, an efficiency map of a generic ceria-based water-splitting reactor is shown in Fig. $1 b$.
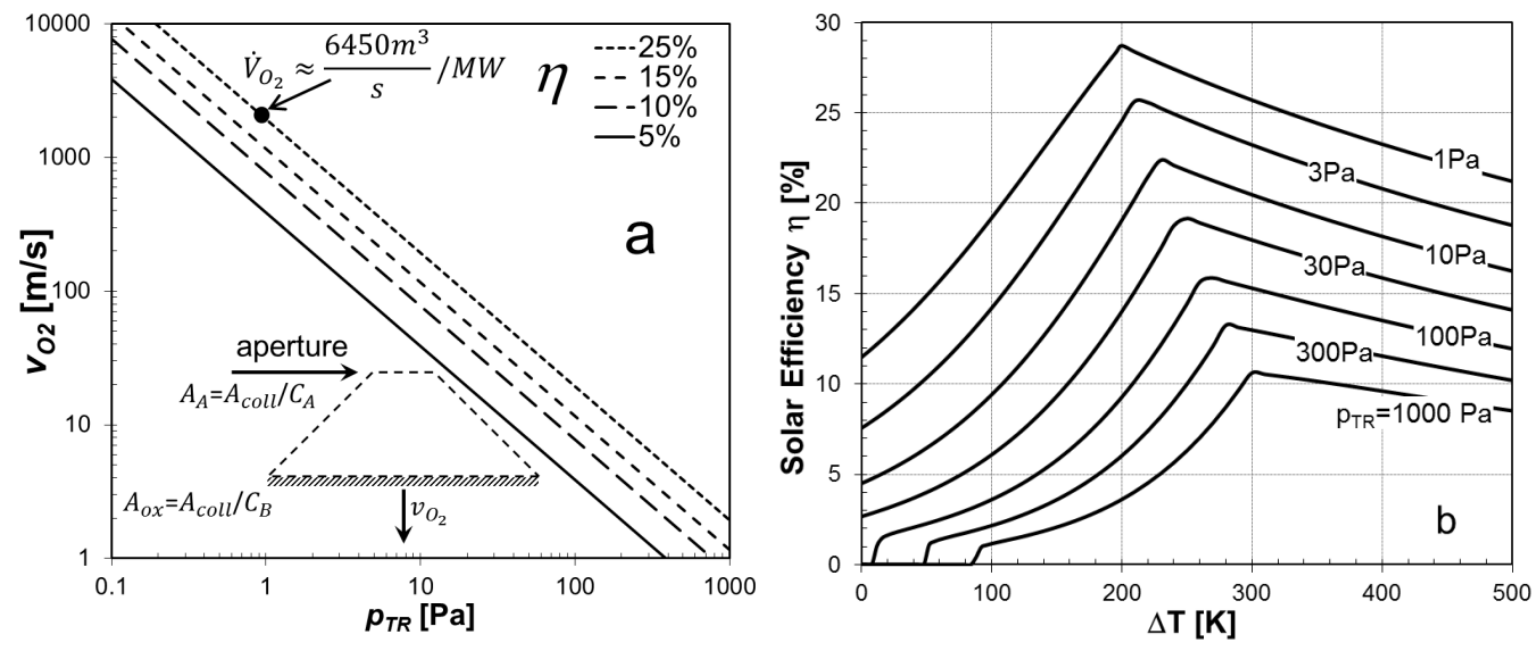

Figure 1. (a) Required oxygen flow velocity from an oxide receiver as function of $p_{T R}, v_{O_{2}}\left(p_{T R}, T_{T R}=1773 \mathrm{~K}\right)$, for solar-to-hydrogen efficiency between $5 \%$ and $25 \%$. One volumetric flow value $-\dot{V}_{O_{2}}(1 P a) \approx \frac{6450 m^{3}}{s} / M W-$ is shown as an example. The lower left inset illustrates flow velocity: sunlight from a concentrator (e.g. a heliostat field) enters an aperture (with area $A_{A}$ ) and illuminates an oxide receiver. Oxygen leaves the oxide at $v_{O_{2}}$. A high concentration $\left(C_{A} \approx 3000\right)$ was assumed, necessary because of the high $T_{T R}$. $C_{B}=300$ (b) Efficiency map for a ceria-based reactor at the same $T_{T R}$, illustrating the efficiency advantage of operating under low $p_{T R}$.[12]

\section{Pressure cascade: efficiency advantages and implementation aspects}

An alternative approach—aimed at decreasing $\dot{V}_{O_{2}}$ and enabling lower $p_{T R}$ limits—consists of performing the thermal reduction step in multiple chambers, each operating at a successively lower pressure. In the cascade concept-illustrated in Fig. $2-\mathrm{O}_{2}$ is pumped near the pressure at which it evolves from the oxide, with only a fraction pumped at the final $p_{T R}$. In the case of a single thermal reduction chamber (previous section), all of the evolved $\mathrm{O}_{2}$ is pumped at the same lowest system pressure.

To estimate $p_{T R}$ limits, a cascade of identically-sized chambers is assumed $\left(A_{o x, i}=A_{o x}\right)$, though $A_{a}$ and $C_{B}$ may differ between chambers. Because using the highest practically achievable $v_{O_{2}}$ (or $\left.\dot{V}_{O_{2}}\right)$ leads to the lowest final $p_{T R}$, a chamber-independent $v_{O_{2}}$ is also assumed $\left(v_{O_{2}, i}=v_{O_{2}}\right)$. From these two assumptions follows $\dot{V}_{O_{2}, i}=\dot{V}_{O_{2}}$. Lastly, the amount of evolved oxygen depends on the extent of reduction, $\delta_{T R}\left(p_{T R}, T_{T R}\right)$ - a property of the oxide itself.

Under the above assumptions, the pressure in the $i^{\text {th }}$ chamber, $p_{T R, i}$, in which the extent of reduction is $\delta_{T R, i}=\delta_{T R}\left(p_{T R, i}\right)$, is given by a recursive relationship:

$$
p_{T R, i}=\dot{n}_{o x} R T_{T R} \frac{\delta_{T R, i}-\delta_{T R, i-1}}{2 \dot{V}_{O_{2}}}
$$

which is simply the ideal gas equation, with the oxygen quantity determined by an oxide molar flow rate $\dot{n}_{o x}$, and an incremental extent of reduction, $\delta_{i n c, i}=\delta_{T R, i}-\delta_{T R, i-1}$. For the first 
chamber (labelled " 0 ") $p_{T R}=p_{T R, O}$ and $\delta_{T R,-1}=\delta_{W S}$. Being a well-characterized candidate material for solar-thermochemical fuel production, $\mathrm{CeO}_{2}$ is assumed as the reactive oxide.[13, 14] Because $\delta\left(p_{T R}, T_{T R}\right)$ for $\mathrm{CeO}_{2}$ follows a complicated relationship, $p_{T R, i}$ is determined numerically for several values of $p_{T R, 0}$, in a relevant pressure range. The results of the calculations are shown in Fig.2.
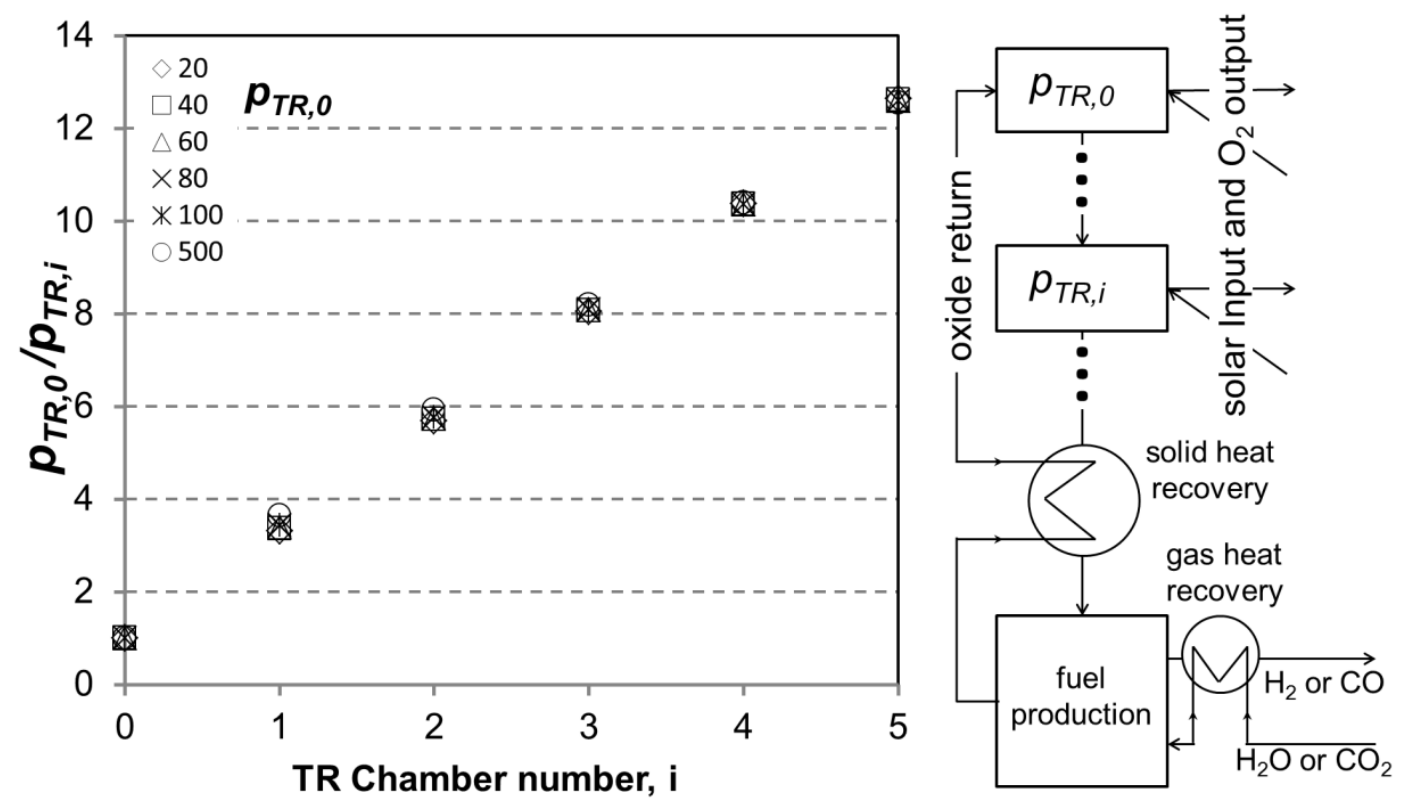

Figure 2. Pumping advantage of a multi-chambered approach. The plot shows the pressure ratio between the $0^{\text {th }}$ and $i^{\text {th }}$ chamber for a ceria-based thermochemical reactor. A simple schematic illustration of a cascading pressure reactor, e.g. associated with a tower-heliostat system, is also shown. Assuming that pressure separation between chambers is accomplished by a reactive oxide in packed particle bed form, isolation valves between chambers were deliberately omitted as reactor components.

The potential for $p_{T R}$ improvement in a cascade is excellent - an order of magnitude $p_{T R}$ decrease can be achieved in as few as five chambers, compared to a single-chambered reactor (Fig.2). The linear and near-identical $p_{T R, O} p_{T R, i}$ relationship for all considered values of $p_{T R, O}$ is particular to ceria. Other materials should be expected to exhibit a different $p_{T R, O} / p_{T R, i}$ dependence. A 10 -fold $p_{T R}$ decrease corresponds to a roughly $7 \%$ absolute solar efficiency increase (Fig.1b).

Since the use of multiple chambers is beneficial, it may seem appealing to assume that arbitrarily many can be used. For example, starting with $p_{T R, 0}=40 \mathrm{~Pa}$, and extending the trend in Fig. 2 to 50 chambers, leads to $p_{T R, 5} \approx 0.35 \mathrm{~Pa}-$ a tempting value from an efficiency perspective! However-because $p_{T R, i} \sim \delta_{i n c, i}$ (eq. 8) - in every subsequent chamber $\delta_{i n c, i}$ decreases, thus limiting number of chambers in practice.

One limit follows from the power per chamber $\left(P_{i}\right)$ : in an approximation in which the enthalpy of oxide reduction, $\Delta H$, is independent of or weakly dependent on $\delta$, a decreasing $\delta_{i n c, i}$ means a decreasing $P_{i} \sim \delta_{i n c, i}$. For example, assuming that heating between $T_{W S}$ and $T_{T R}$ is accomplished prior to thermal reduction, and that the solar input power in the $0^{\text {th }}$ chamber is $P_{0}=3 \mathrm{MW}$ (used entirely for thermal reduction), then $P_{5}$ would be a reasonable $235 \mathrm{~kW}$, but $P_{50}$ would be only $26 \mathrm{~kW}$. Delivering such low power to into a chamber through a finite aperture (at 
$C_{A} \approx 3000$ ) would be challenging in a tower-heliostat configuration, even if mitigation methods are devised.

The finite angular diameter of the Sun $(\phi \approx 9 \mathrm{mrad})$ and windowed-aperture size limits $\left(A_{A} \approx 1 \mathrm{~m}^{2}\right)$, for example, limit the heliostat-aperture distance to $\approx 110 \mathrm{~m}$, constraining tower heights and heliostat field sizes. Costs associated with a large number of chambers, each additional one bringing a diminishing efficiency benefit, should also be considered.

Irrespective of limitations, the above results indicate that at least a 10 to 20 -fold $p_{T R}$ decrease can be achievedin a pressure cascade - bringing the minimum practical $p_{T R}$ to $1 \mathrm{~Pa}$ or less. Under realistic operating circumstances, the resulting $\delta_{T R}$ increase can lead to a substantial efficiency advantage compared to equivalent single-chambered designs (Fig.1b).

To maintain a pressure difference between neighboring chambers, oxygen flow between them must be negligible. This requirement means that the flow between the $\mathrm{i}^{\mathrm{th}}$ and the preceding chamber, at pressures $p_{T R, i}$ and $p_{T R, i-1}$, should be $\dot{V}_{i, i-1}<<\dot{V}_{O_{2}}$.

One possible approach, placing "airlocks" between chambers, i.e. double valves that open asynchronously, would allow a quasi-continuous oxide flow and reactor operation, while restricting oxygen cross-flow. Unfortunately, airlocks are unlikely to be viable pressure separation devices in the hostile operating environment of solar-thermochemical reactors.

A more practical pressure separation method is one in which the reactive oxide is in the form of a moving packed bed, building on reactor the design described in [7]. In this design, the oxide bed itself acts as a pressure separation agent between chambers, owing to the low gas permeability of packed particle beds, governed by Darcy's law. Compared to free HagenPoiseuille flow, the flow restriction factor depends on the bed permeability, and is $>10^{6}$ under conditions expected in a reactor for thermochemical fuel production.[15]

In this approach, pressure separation between two chambers can be quantified via the volumetric flow through a packed bed:

$$
\dot{V}_{i, i-1}=\frac{k A \Delta p_{T R, i}}{\mu L}
$$

Here, $k \approx 10^{-11} \mathrm{~m}^{2}$ is the bed permeability (typical for fine to medium-sized particulate materials), and $\mu \approx 10^{-4} \mathrm{~Pa} \mathrm{~s}$ is the gas dynamic viscosity. For a typical $p_{T R, 0}<100 \mathrm{~Pa}$, the pressure difference between chambers can be taken as $\Delta p_{T R, i} \approx 25 \mathrm{~Pa}$. $L \approx 1 \mathrm{~m}$ and $A \approx 100 \mathrm{~cm}^{2}$ are the length and crosssection area of a connection between chambers.

Under these assumptions, $\dot{V}_{i, i-1} \approx 3 * 10^{-8} \mathrm{~m}^{3} / \mathrm{s}$ - a value vastly smaller than the expected $\dot{V}_{O_{2}} \approx \frac{100 m^{3}}{s} / M W$ (power normalization with respect to $P_{S}$ ). This exceptional flow restriction means that oxygen exchange between chambers would effectively consist only of the gas in the bed void fraction $\left(\dot{V}_{\text {void }}\right)$, itself proportional to the oxide volumetric flow $\dot{V}_{O X}$. For a well-performing oxide $(\Delta \delta \approx 0.2)$ with a reasonable molar mass $\left(M_{o x} \sim 0.2 \mathrm{~kg} / \mathrm{mol}\right)$, a bed density $\left(\rho=4000 \mathrm{~kg} / \mathrm{m}^{3}\right)$, and a void fraction $\phi=50 \%, \dot{V}_{\text {void }}=\frac{\dot{n}_{H_{2}} M_{O X^{\Phi}}}{\rho \Delta \delta} \approx \frac{10^{-4} \mathrm{~m}^{3}}{\mathrm{~s}} / M W$-also vastly smaller than $\dot{V}_{O_{2}}$. These results give confidence that pressure separation between chambers in a cascade can be accomplished under all imaginable practical circumstances, including a wide range of particle sizes.

\section{Conclusions}

Large required $\mathrm{O}_{2}$ volumetric flows and velocities limit the lowest practical achievable pressure in the thermal reduction step of vacuum-pumped, two-step thermochemical cycles for 
solar fuel production. These limits can be substantially mitigated by the use of a cascade of thermal reduction chambers, each operating at a successively lower pressure. A reactor design based on a moving packed bed of reactive oxide particles can accomplish the necessary pressure separation between thermal reduction chambers without requiring the use of high-temperature airlocks. The cascade approach has the potential to decrease the thermal reduction pressure limit by over an order of magnitude, to below $1 \mathrm{~Pa}$, yielding significant efficiency gains.

\section{Acknowledgements}

This work was supported by the U.S. Department of Energy Fuel Cell Technologies Program via the Solar Thermochemical Hydrogen $(\mathrm{STCH})$ directive. Sandia is a multiprogram laboratory operated by Sandia Corporation, a Lockheed Martin Company, for the United States Department of Energy's National Nuclear Security Administration under Contract DE-AC04-94AL85000.

\section{References}

[1] Fletcher, E. A., and Moen, R. L., 1977, "Hydrogen and Oxygen from Water", Science, 197(4308), 10501056.

[2] Nakamura, T., 1977, "Hydrogen Production from Water Utilizing Solar Heat at High Temperatures", Solar Energy, 19(5), 467-475.

[3] Funk, J. E., and Reinstrom, R. M., 1966, "Energy Requirements in the Production of Hydrogen from Water", Industrial \& Engineering Chemistry Process Design, 5(3), 336-342.

[4] Steinfeld, A., Sanders, S., and Palumbo, R., 1999, "Design Aspects of Solar Thermochemical

Engineering - a Case Study: Two-Step Water-Splitting Cycle Using the $\mathrm{Fe}_{3} \mathrm{O}_{4} /$ Feo Redox System", Solar Energy, 65(1), 43-53.

[5] Diver, R. B., Miller, J. E., Allendorf, M. D., Siegel, N. P., and Hogan, R. E., 2008, "Solar Thermochemical Water-Splitting Ferrite-Cycle Heat Engines", Journal of Solar Energy Engineering, 130(4), 041001-1 041001-8.

[6] Nrel, 18.Nov.2011, http://rredc.nrel.gov/solar/old data/nsrdb/1961-1990/redbook/atlas/

[7] Ermanoski, I., Siegel, N. P., and Stechel, E. B., 2013, "A New Reactor Concept for Efficient Solar-

Thermochemical Fuel Production", Journal of Solar Energy Engineering, 135(3), 031002-1 - 10.

[8] Chueh, W. C., Falter, C., Abbot, M., Scipio, D., Furler, P., Haile, S., and Steinfeld, A., 2010, "High-Flux Solar-Driven Thermochemical Dissociation of $\mathrm{CO}_{2}$ and $\mathrm{H}_{2} \mathrm{O}$ Using Nonstoichiometric Ceria", Science, 330(6012), 1797-1800.

[9] Lapp, J., Davidson, J. H., and Lipinski, W., 2012, "Efficiency of Two-Step Solar Thermochemical NonStoichiometric Redox Cycles with Heat Recovery", Energy, 37(1), 591-600.

[10] Bader, R., Venstrom, L. J., Davidson, J. H., and Lipiński, W., 2013, "Thermodynamic Analysis of Isothermal Redox Cycling of Ceria for Solar Fuel Production", Energy \& Fuels, 27(9), 5533-5544.

[11] Hao, Y., Yang, C.-K., and Haile, S. M., 2013, "High-Temperature Isothermal Chemical Cycling for Solar-Driven Fuel Production", Physical Chemistry Chemical Physics, 15(17084-17092.

[12] Ermanoski, I., Miller, J. E., and Allendorf, M. D., 2014, "Efficiency Maximization in SolarThermochemical Fuel Production: Challenging the Concept of Isothermal Water Splitting", Physical Chemistry Chemical Physics, 16(18), 8418-8427.

[13] Panlener, R. J., Blumenthal, R. N., and Garnier, J. E., 1975, "A Thermodynamic Study of Nonstoichiometric Cerium Dioxide", Journal of Physics and Chemistry of Solids, 36(11), 1213-1222.

[14] Zinkevich, M., Djurovic, D., and Aldinger, F., 2006, "Thermodynamic Modelling of the CeriumOxygen System", Solid State lonics, 177(11-12), 989-1001.

[15] Darcy, H., 1856, Les Fontaines Publiques De La Ville De Dijon, Paris. 